\title{
BMJ Open Health science communication strategies used by researchers with the public in the digital and social media ecosystem: a systematic scoping review protocol
}

\author{
Guillaume Fontaine, ${ }^{1,2}$ Andréane Lavallée, ${ }^{1,3}$ Marc-André Maheu-Cadotte,, ${ }^{1,2,4}$ \\ Julien Bouix-Picasso, ${ }^{1,5}$ Anne Bourbonnais ${ }^{1,6}$
}

To cite: Fontaine G, Lavallée A, Maheu-Cadotte M-A, et al. Health science communication strategies used by researchers with the public in the digital and social media ecosystem: a systematic scoping review protocol. BMJ Open 2018;8:e019833. doi:10.1136/ bmjopen-2017-019833

- Prepublication history and additional material for this paper are available online. To view these files, please visit the journal online (http://dx.doi. org/10.1136/bmjopen-2017019833).

Received 27 September 2017 Revised 8 December 2017 Accepted 11 December 2017

Check for updates

For numbered affiliations see end of article.

Correspondence to Guillaume Fontaine; guillaume.fontaine@umontreal. ca

\section{ABSTRACT}

Introduction The optimisation of health science communication (HSC) between researchers and the public is crucial. In the last decade, the rise of the digital and social media ecosystem allowed for the disintermediation of HSC. Disintermediation refers to the public's direct access to information from researchers about health science-related topics through the digital and social media ecosystem, a process that would otherwise require a human mediator, such as a journalist. Therefore, the primary aim of this scoping review is to describe the nature and the extent of the literature regarding HSC strategies involving disintermediation used by researchers with the public in the digital and social media ecosystem. The secondary aim is to describe the HSC strategies used by researchers, and the communication channels associated with these strategies.

Methods and analysis We will conduct a scoping review based on the Joanna Briggs Institute's methodology and perform a systematic search of six bibliographical databases (CINAHL, EMBASE, IBSS, PubMed, Sociological Abstracts and Web of Science), four trial registries and relevant sources of grey literature. Relevant journals and reference lists of included records will be hand-searched. Data will be managed using the EndNote software and the Rayyan web application. Two review team members will perform independently the screening process as well as the full-text assessment of included records. Descriptive data will be synthesised in a tabular format. Data regarding the nature and the extent of the literature, the HSC strategies and the associated communication channels will be presented narratively.

Ethics and dissemination This review does not require institutional review board approval as we will use only collected and published data. Results will allow the mapping of the literature about HSC between researchers and the public in the digital and social media ecosystem, and will be published in a peer-reviewed journal.

\section{INTRODUCTION}

The optimisation of health science communication (HSC) between researchers and the public is crucial. Indeed, the public's understanding of health science can be influential

\section{Strengths and limitations of this study}

This scoping review protocol is based on a rigorous and systematic methodology.

- The search strategy was built in collaboration with a librarian experienced in science communication to identify research foci and knowledge gaps within the last 18 years.

- No quality assessment or risk of bias assessment are planned regarding the included literature, as recommended by the Joanna Briggs Institute scoping review methodology.

- This review will be limited to the literature published in English or French.

to governmental decision-making, health policies, health promotion, disease prevention and self-care. ${ }^{1-3}$ The direct relationship between researchers and the public is an emerging dimension in the literature about HSC and the public's understanding of science. The field of HSC has multiple stakeholders including, among others, researchers, scientific societies, scientific organisations, universities, government agencies, news and media organisations and public information officers. ${ }^{45}$ Moreover, HSC falls within the broader domain of the mass communication of scientific and biomedical evidence including theoretical constructs and findings related to the news and mass media's impact, health communication interventions, numeracy and health literacy. ${ }^{4} 6$

Over the last decade, the digital and social media ecosystem has reshaped the concept of HSC by enabling new, direct and powerful communication channels between researchers and the public. ${ }^{7}$ Nearly $70 \%$ of the public looking for information about specific science issues cite the Internet as their primary source of information. ${ }^{8}$ Internet based and interactive communication 
channels, such as social media and content-sharing platforms, allow for the disintermediation of HSC between researchers and the public. Disintermediation refers to the public's direct access to information from researchers about health science-related topics through the social and digital media ecosystem, a process that would otherwise require a human mediator, such as a journalist. ${ }^{9}$ Indeed, before the rise of the digital media ecosystem, HSC was mainly a process involving researchers (specialist discourse), institutions (didactic explanation) and journalists (popularisation). Experts now suggest that there is a contemporary public expectation for researchers to deliver HSC themselves. ${ }^{10}$

Enablers of this new paradigm of HSC, in which researchers play a forefront role, are steadily increasing in the digital and social media ecosystem. Online resources such as the Science Media Centre (www.sciencemediacentre. org) and The Conversation (www.theconversation.com) aim to maintain a free flow of information from knowledge creation to knowledge dissemination. Social media platforms, the largest in the Anglosphere being Facebook, Instagram, Twitter, Snapchat and LinkedIn, are seeing a growing influx of users. ${ }^{11-13}$ Content-sharing platforms, such as YouTube and Reddit, are attracting together more than 2 billion monthly visitors, and offer many types of content, including amateur and professional HSC. ${ }^{14}{ }^{15}$ Thus, through these platforms, information can be relayed to millions in a matter of minutes and induce, amplify or subdue individuals' responses regarding health science-related issues. ${ }^{16}$ Over recent years, we have seen evidence that these new communication channels can empower the public to effectively organise and reshape local, national and international policies related to health science. For instance, the 'March for Science', held in more than 600 cities across the world on Earth Day, 22 April 2017, originated from a discussion on Reddit. ${ }^{1718}$ Meanwhile, we have seen new conceptions of HSC emerge, which are collaborative, democratic and deliberative in nature, to establish a direct and reciprocal dialogue between researchers and the public. ${ }^{19}$

In light of the disintermediation of HSC and the growing influence of the digital and social media ecosystem on the public, a recent US National Academies of Sciences, Engineering and Medicine report suggests researchers should strive to develop and implement novel HSC strategies to boost citizen engagement in health science-related topics. ${ }^{4}$ More specifically, the Academies highlighted the need for the aggregation of information from studies so an evidence base can be built to 'help science communicators determine which approaches to communicating are effective for whom and under what conditions for achieving specific communication goals'. These knowledge synthesis efforts should consider the disintermediation of HSC between researchers and the public, the growing influence of the digital and social media ecosystem on the public, and the collaborative, democratic and deliberative nature of recent HSC conceptions. Thus, the primary aim of this scoping review is to describe the nature and the extent of the literature regarding HSC strategies involving disintermediation used by researchers with the public in the digital and social media ecosystem. The secondary aim of this scoping review is to describe the HSC strategies involving disintermediation used by researchers with the public, as well as the communication channels associated with these strategies.

A scoping review is defined as a subtype of systematic review aiming to map the available evidence related to key concepts underpinning a topic area, to identify gaps in the evidence and report on the types of evidence that address and inform practice. More precisely, 'scoping reviews can be used to map evidence in relation to time (when it was published), location (country), source (peer reviewed or grey literature), and/or origin (healthcare or academic discipline) ${ }^{20}$ Before undertaking this scoping review, relevant databases were searched to identify previous reviews addressing the same objective and none were identified.

\section{Scoping review questions}

This scoping review aims to address the following questions:

1. What is the nature and the extent of the literature regarding the HSC strategies involving disintermediation used by researchers with the public in the digital and social media ecosystem?

2. What are the characteristics, the barriers and the facilitators of HSC strategies involving disintermediation used by researchers with the public in the digital and social media ecosystem?

3. What are the communication channels associated with these HSC strategies?

\section{METHODS}

This scoping review protocol is based on the methodology proposed by the Joanna Briggs Institute (JBI) ${ }^{20}$ This methodology integrates the methodological frameworks proposed by Arksey and $\mathrm{O}^{\prime}$ Malley ${ }^{21}$ and Levac et $a l .{ }^{22}$ To ensure a systematic approach and the inclusion of all relevant methodological aspects, the protocol is based on the Preferred Reporting Items for Systematic Review and Meta-Analysis Protocols (PRISMA-P) Checklist ${ }^{23}$ (see online supplementary file 1 ). Since the aim of a scoping review is not to provide a comprehensive assessment of the quality of included literature, the items 14, 15, 16 and 17 of the PRISMA-P Checklist are not reported. Furthermore, as recommended by JBI manual, ${ }^{20}$ we structured our protocol by Population-Concept-Context instead of Population-Intervention-Comparator-Outcomes, as suggested in PRISMA-P.

\section{Inclusion criteria}

Population

We will consider the literature reporting HSC strategies involving disintermediation used by researchers in health disciplines (first population) with the general public (second population). 
Table 1 Conceptions of science communication

\begin{tabular}{lll}
\hline Conception & Definition \\
\hline Type 1 & $\begin{array}{l}\text { Professional science } \\
\text { communication }\end{array}$ & $\begin{array}{l}\text { Knowledge exchanged among researchers; associated with the professional practice of } \\
\text { science. }\end{array}$ \\
Type 2 & $\begin{array}{l}\text { Deficit science } \\
\text { communication }\end{array}$ & $\begin{array}{l}\text { Knowledge exchanged from researchers to the public. } \\
\text { Type } 3\end{array}$ \\
$\begin{array}{l}\text { Consultative science } \\
\text { communication }\end{array}$ & $\begin{array}{l}\text { Knowledge exchanged iteratively from researchers to the public, and from the public to } \\
\text { the researchers. }\end{array}$ \\
Type 4 & $\begin{array}{l}\text { Deliberative science } \\
\text { communication }\end{array}$ & $\begin{array}{l}\text { Knowledge exchanged in a democratic and deliberative manner in which the principal } \\
\text { actors have equal standing, and scientific knowledge and local knowledge are mutually } \\
\text { respected. }\end{array}$ \\
\hline
\end{tabular}

We will consider for inclusion literature about researchers in all health disciplines, as defined by the classification of the WHO. ${ }^{24}$ Examples of health disciplines that will be included are medicine, dentistry, midwifery, pharmacy, psychology, and nursing. We will consider for inclusion literature involving the general public, or specific sociodemographic groups at large (eg, teenagers, young adults and women). However, we will exclude literature involving specifically patients and students, since other fields of study relate more directly to these populations (eg, patient education and health sciences education).

\section{Concept}

The central concept of this scoping review is HSC. Various conceptions of science communication, in general, are proposed by researchers throughout the world. A recent typology summarised four conceptions of science communication (see table 1). Aside from professional science communication (type 1 ), which is embedded in the professional practice of science, deficit science communication (type 2) is the most common type of science communication across the world. ${ }^{19}$ This is reflected by the fact that nearly all Anglo-Saxons funding agencies started promoting such type of science communication at the beginning of the 21st century. However, Palmer and Schibeci demonstrated that funding agencies have begun to promote consultative and deliberative forms of science communication (types 3 and 4) in the last decade, indicating a global policy shift to engage the public in science. ${ }^{19}$ In the context of this review, we adopted a broad definition of HSC that encompasses all conceptions of science communication between researchers and the public (types 2, 3 and 4). We define HSC as an interactive process of knowledge exchange between researchers and the public involving the use of communication strategies (psychosocial means) through various communication channels (digital and physical means).

Further, we define a HSC strategy involving disintermediation as any plan or action adopted by researchers (eg, using humour, vulgarising research findings and telling a story), ${ }^{25}$ operationalised through various communication channels without a human mediator in the digital and social media ecosystem (eg, social media, content-sharing platforms, podcasts and blog). This definition implies that we will not consider for inclusion HSC strategies implemented in faceto-face, ie a researcher interacting with a non-expert audience. Moreover, it should be noted that strategies used by researchers to directly influence policy-making or political instances are beyond the scope of this review.

\section{Context}

We will consider for inclusion sources reporting HSC strategies involving disintermediation used by researchers with the public about any topic areas related to clinical aspects of health. Examples of topic areas which will be considered for inclusion are vaccination, mental health, sexual health and cardiovascular health. However, we will exclude topic areas that are not directly related to clinical aspects of health, such as climate change, employment and economics.

\section{Types of records}

We will consider records published in English or in French, regardless of the geographic location. Since HSC disintermediation is a concept strongly related to the rise of the digital and social media ecosystem in the last two decades, ${ }^{79} 10$ more particularly social media and contentsharing platforms, we will search the literature between 2000 and 2018.

We will consider primary studies with any design: quasi-experimental designs (before and after, and interrupted time series) and experimental designs (randomised controlled trials), observational designs (cohort, casecontrol and cross-sectional), qualitative designs and mixed-methods designs. We will also examine grey literature (theses and dissertations, government reports, policy statements, research reports and conference proceedings), opinion papers and knowledge synthesis papers.

\section{Literature search}

\section{Information sources}

\section{Bibliographical databases}

We will identify eligible records through a comprehensive literature search of six bibliographic databases: Cumulative Index to Nursing and Allied Health Literature (CINAHL), via EBSCO; Excerpta Medica dataBASE (EMBASE), via OVID; International Bibliography of the Social Sciences (IBSS), via ProQuest; PubMed, 
via NCBI; Sociological Abstracts, via ProQuest); Web of Science-Science Citation Index (SCI) and Social Sciences Citation Index (SSCI), , via ISI-Thomson Scientific.

\section{Grey literature and additional sources}

We will perform a comprehensive search of relevant grey literature sources: Campbell Collaboration Systematic Reviews, Cochrane Database of Systematic Reviews, Grey Guide, Joanna Briggs Institute, Open Grey and ProQuest Dissertation and Theses. We will also search trial registries for relevant records (www.ClinicalTrials.gov; www.clinicalt rialsregister.eu; www.isrctn.com; www.anzctr.org.au).

\section{Hand-searching}

We will hand-search relevant journals to identify additional records. Examples of sources include journals such as: Communication Research, Health Communication, Journal of Health Communication, Journalism and Mass Communication Quarterly, Public Understanding of Science and Science Communication.

\section{Reference searching}

Using a snowball method, we will screen the reference list of included records to identify additional records.

\section{Search strategy}

We developed the search strategy for this scoping review in collaboration with a librarian experienced in science communication. The search strategy uses a combination of three major concepts (see table 2): (1) researchers and the public; (2) HSC; (3) disintermediation, which refers to the concept in itself, as well as to the communication channels in the digital and social media ecosystem. We first developed the search strategy for PubMed (see online supplementary file 2), and then translated it for other databases. The search strategy was refined over a period of 2 months to ensure replicability in all databases. We plan to perform the search of relevant keywords in titles and abstracts. Searching in titles and abstracts allows us to retrieve recent publications not yet indexed with MeSH terms.

\section{Study records}

Data management

Records obtained with the search strategy will be managed using the EndNote X8 software (V8.0.1., Clarivate Analytics). Duplicates will be deleted and references exported in the Rayyan web application (Qatar Computing Research Institute) to facilitate independent record selection by the scoping review team. ${ }^{26}$

\section{Selection process}

Two review team members will independently screen the titles and abstracts of records retrieved by the search strategy and apply the eligibility criteria. Two review team members will then perform independently

Table 2 Major concepts and related keywords used for building the search strategy

\begin{tabular}{|c|c|c|c|c|c|}
\hline \multicolumn{2}{|l|}{ Concept 1} & \multicolumn{3}{|l|}{ Concept 2} & \multirow{2}{*}{$\begin{array}{l}\text { Concept } 3 \\
\text { Disintermediation }\end{array}$} \\
\hline Researchers & Public & Health & Science & Communication & \\
\hline Researcher* & Public & Biomedical & Science* & Communicati* $^{*}$ & Internet \\
\hline Scientist $^{*}$ & Non-experts & Medical & Research & Disseminati $^{\star}$ & Online \\
\hline Expert* & Population & & Scholarly & Populariz ${ }^{*}$ & Digital \\
\hline \multirow[t]{17}{*}{ Investigator } & & & Findings & Vulgariz ${ }^{*}$ & Web* \\
\hline & & & Results & Diffusion & Interactive \\
\hline & & & Innovation* & Outreach & Social media \\
\hline & & & Evidence & & YouTube \\
\hline & & & & & Twitter \\
\hline & & & & & Facebook \\
\hline & & & & & Reddit \\
\hline & & & & & Instagram \\
\hline & & & & & Vimeo \\
\hline & & & & & Linkedln \\
\hline & & & & & Podcasts \\
\hline & & & & & iTunes U \\
\hline & & & & & Forum \\
\hline & & & & & Discussion board \\
\hline & & & & & Content sharing \\
\hline & & & & & Video sharing \\
\hline & & & & & Blog \\
\hline
\end{tabular}


the full-text assessment of included records. At any time during the review process, disagreements will be resolved through discussion and consensus. A third author will be involved in case of a persistent disagreement. We will document reasons for exclusion at the full-text phase of the screening process. A unique identification number will be attributed to included records for data extraction.

\section{Data extraction process}

Two review team members (AB and M-AM-C) developed two grids to facilitate data extraction from included records. One grid was developed specifically for original research articles and all types of systematic reviews and the other one was developed for other types of articles (eg, opinion paper and editorial). These grids were pilot tested and iteratively validated by the whole team to insure their completeness and clarity.

\section{Data items}

Using the two data extraction grids developed for this scoping review, we will extract the following data from included records:

1. Descriptive data: year of publication, first author's academic discipline, country of origin, article type (eg, study, editorial and review) and aim.

2. Theoretical data: theoretical framework, if mentioned.

3. Methodological data: study design (eg, interrupted time series and metasynthesis), population and sample size (if applicable), data collection and analysis method and article limits (according to the authors).

4. Data on HSC strategies and communication channels:

A. characteristics, barriers and facilitators of HSC strategies involving disintermediation in the digital and social media ecosystem.

B. communication channels associated with HSC strategies involving disintermediation in the digital and social media ecosystem.

5. Results data: reported results according to study outcomes.

\section{Data synthesis}

We will present a PRISMA diagram to depict the flow of considered records during the screening process. ${ }^{27}$ Descriptive, theoretical, methodological and results data will be synthesised in a tabular form. Results regarding HSC strategies involving disintermediation used by researchers with the public, and the communication channels associated with these strategies, will be presented through a narrative synthesis.

\section{DISCUSSION}

The purpose of this scoping review is to describe the nature and the extent of the literature regarding HSC strategies involving disintermediation used by researchers with the public in the digital and social media ecosystem.
This is crucial in an era in which researchers must reinforce the role of health science in public decision-making in response to global, national and local health issues. We believe the results will be theoretically relevant to researchers, academics and policy-makers in order to optimise HSC with the public.

The limits of this scoping review include no planned quality assessment of records, as recommended in methodological frameworks, which can hinder the recommendations for practice. Moreover, synthesising the results of various information sources can often be challenging. The strengths of this scoping review include the adoption of a systematic approach, the adherence to a rigorous and recognised methodological framework and the inclusion of a wide variety of information sources. The review team is composed of doctoral students experienced in the field of HSC working in close collaboration with an experienced professor and a librarian.

Author affiliations

${ }^{1}$ Faculty of Nursing, Université de Montréal, Montreal, Québec, Canada ${ }^{2}$ Research Center, Montreal Heart Institute, Montréal, Quebec, Canada ${ }^{3}$ Research Center, CHU Sainte-Justine, Montreal, Quebec, Canada

${ }^{4}$ Research Center, Centre hospitalier de l'Université de Montréal, Montreal, Québec, Canada

${ }^{5}$ Laboratoire Educations et Pratiques en Santé (EA 3412), Service santé des armées, Université Paris 13 Ecole du Val-de-Grâce, Paris, Île-de-France, France

${ }^{6}$ Research Center, Institut Universitaire de Gériatrie de Montréal, Montreal, Québec, Canada

Acknowledgements We would like to acknowledge the participation of Lysane Paquette, R.N., M.Sc., and Natalie Stake-Doucet, R.N., M.Sc. (A), in the early stages of this scoping review protocol. We would also like to acknowledge the contribution of Hélène Tardif, librarian at the Université de Montréal, who helped build the search strategy. GF wishes to thank Quebec's Healthcare Research Fund, Quebec's Ministry of Higher Education, the Canadian Nurses Foundation, the Montreal Heart Institute Foundation and the Faculty of Nursing at the Université de Montréal for the financial support they provided for his doctoral studies. AL would like to acknowledge the CHU Sainte-Justine, the Committee for Nursing Research Development, TD Bank and Quebec's Ministry of Higher Education for their financial support for her doctoral studies. M-AM-C would like to acknowledge the scholarship received from the followings: Montreal Heart Institute Foundation, Quebec's Ministry of Higher Education, Faculty of Nursing of the Université de Montréal and the Fondation J.A. DeSève. JB-P would like to acknowledge the Liliane Martel doctoral grant of the Chaire de recherche sur les nouvelles pratiques de soins infirmiers of Université de Montréal and Service de santé des armées for their financial support for his doctoral studies.

Contributors GF, AL and M-AM-C drafted the initial search strategy. JB-P and AB refined and validated the search strategy. All authors contributed to protocol writing. All authors read and approved the final version of the manuscript. All authors will contribute to title and abstract screening, full-text assessment of records, data extraction and data synthesis.

Funding This research received no specific grant from any funding agency in the public, commercial or not-for-profit sectors.

Competing interests None declared.

Patient consent Not required.

Provenance and peer review Not commissioned; externally peer reviewed.

Open Access This is an Open Access article distributed in accordance with the Creative Commons Attribution Non Commercial (CC BY-NC 4.0) license, which permits others to distribute, remix, adapt, build upon this work non-commercially, and license their derivative works on different terms, provided the original work is properly cited and the use is non-commercial. See: http://creativecommons.org/ licenses/by-nc/4.0/ 
(c) Article author(s) (or their employer(s) unless otherwise stated in the text of the article) 2018. All rights reserved. No commercial use is permitted unless otherwise expressly granted.

\section{REFERENCES}

1. Stilgoe J, Lock SJ, Wilsdon J. Why should we promote public engagement with science? Public Underst Sci 2014;23:4-15.

2. Meisel ZF, Gollust SE, Grande D. Translating research for health policy decisions: is it time for researchers to join social media? Acad Med 2016;91:1341-3.

3. Richardson DP. Consumer communication of nutrition science and impact on public health. Science and the law: how the communication of science affects policy development in the environment, food, health, and transport sectors. Washington, DC: American Chemical Society, 2015:29-46.

4. National Academies of Sciences Engineering, and Medicine. Communicating science effectively: a research agenda. Washington, DC: The National Academies Press, 2017.

5. Marcinkowski F, Kohring M. The changing rationale of science communication: a challenge to scientific autonomy. JCOM 2014;13:C04.

6. Logan RA, Siegel E. Health literacy: new directions in research, theory, and practice. Amsterdam, Netherlands: IOS Press, 2017.

7. Brownell SE, Price JV, Steinman L. Science communication to the general public: why we need to teach undergraduate and graduate students this skill as part of their formal scientific training. J Undergrad Neurosci Educ 2013;12:E6-E10.

8. National Science Board. Science and engineering indicators 2016. Washington, DC: National Science Board, 2016.

9. Gerber A. Reinventing science journalism in the age of disintermediation. 4th European Conference for Science Journalists. Copenhagen, Denmark, 2017.

10. Gerber A. Science caught flat-footed: how academia struggles with open science communication. In: Sn B, Friesike S, eds. Opening science: the evolving guide on how the internet is changing research, collaboration and scholarly publishing. Heidelberg, Germany: Springer Open, 2014:73-80.

11. Golding P, Raeymaeckers K, Sousa H. Social media - new challenges and approaches for communications research. Eur $J$ Commun 2017;32:3-5.
12. Schnitzler K, Davies N, Ross F, et al. Using Twitter ${ }^{\mathrm{TM}}$ to drive research impact: a discussion of strategies, opportunities and challenges. Int $\mathrm{J}$ Nurs Stud 2016;59:15-26.

13. Haustein $S$, Sugimoto $C$, Larivière V. Guest editorial: social media in scholarly communication. Aslib Journal of Information Management 2015;67:246-59.

14. Welbourne DJ, Grant WJ. Science communication on youtube: factors that affect channel and video popularity. Public Underst Sci 2016;25:706-18.

15. Kloepper L, Pyzdek A. Reddit Science AMA: Using "the front page of the internet" to share your knowledge with the world!. J Acoust Soc Am 2015;137:2367-67.

16. Fung IC, Tse ZT, Cheung $\mathrm{CN}$, et al. Ebola and the social media. Lancet 2014;384:2207.

17. Guarino B. The March for Science began with this person's 'throwaway line' on Reddit. Washington, DC: Washington Post 2017.

18. Kaplan S. Are scientists going to march on Washington? Washington, DC: Washington Post, 2017.

19. Palmer SE, Schibeci RA. What conceptions of science communication are espoused by science research funding bodies? Public Underst Sci 2014;23:511-27.

20. The Joanna Briggs Institute. Joanna Briggs Institute reviewers' manual: 2015 edition / supplement. Adelaide, Australia: The Joanna Briggs Institute, 2015

21. Arksey H, O'Malley L. Scoping studies: towards a methodological framework. Int J Soc Res Methodol 2005;8:19-32.

22. Levac D, Colquhoun H, O'Brien KK. Scoping studies: advancing the methodology. Implement Sci 2010;5:69.

23. Shamseer L, Moher D, Clarke M, et al. Preferred reporting items for systematic review and meta-analysis protocols (PRISMA-P) 2015 elaboration and explanation. BMJ 2015;349:g7647.

24. World Health Organization. International classification of health workers. Geneva, Switzerland: World Health Organization, 2010.

25. Heath C, Heath D. Made to stick: why some ideas survive and others die. New York, NY: Random House, 2007.

26. Ouzzani M, Hammady H, Fedorowicz Z, et al. Rayyan-a web and mobile app for systematic reviews. Syst Rev 2016;5:1-10.

27. Moher D, Liberati A, Tetzlaff J, et al. Preferred reporting items for systematic reviews and meta-analyses: the PRISMA statement. PLoS Med 2009;6:e1000097. 